\title{
Mutation in mitochondrial ribosomal protein MRPS22 leads to Cornelia de Lange-like phenotype, brain abnormalities and hypertrophic cardiomyopathy
}

\author{
Paulien Smits ${ }^{1,7}$, Ann Saada ${ }^{2,7}$, Saskia B Wortmann ${ }^{1}$, Angelien J Heister ${ }^{3}$, Maaike Brink ${ }^{1}$, Rolph Pfundt ${ }^{3}$, \\ Chaya Miller ${ }^{2}$, Dorothea Haas ${ }^{4}$, Ralph Hantschmann ${ }^{5}$, Richard JT Rodenburg ${ }^{1}$, Jan AM Smeitink ${ }^{1}$ and \\ Lambert $P$ van den Heuvel ${ }^{\star, 1,6}$
}

The oxidative phosphorylation (OXPHOS) system is under control of both the mitochondrial and the nuclear genomes; 13 subunits are synthesized by the mitochondrial translation machinery. We report a patient with Cornelia de Lange-like dysmorphic features, brain abnormalities and hypertrophic cardiomyopathy, and studied the genetic defect responsible for the combined OXPHOS complex I, III and IV deficiency observed in fibroblasts. The combination of deficiencies suggested a primary defect associated with the synthesis of mitochondrially encoded OXPHOS subunits. Analysis of mitochondrial protein synthesis revealed a marked impairment in mitochondrial translation. Homozygosity mapping and sequence analysis of candidate genes revealed a homozygous mutation in MRPS22, a gene encoding a mitochondrial ribosomal small subunit protein. The mutation predicts a Leu215Pro substitution at an evolutionary conserved site. Mutations in genes implicated in Cornelia de Lange syndrome or copy number variations were not found. Transfection of patient fibroblasts, in which MRPS22 was undetectable, with the wild-type MRPS22 cDNA restored the amount and activity of OXPHOS complex IV, as well as the 12S rRNA transcript level to normal values. These findings demonstrate the pathogenicity of the MRPS22 mutation and stress the significance of mutations in nuclear genes, including genes that have no counterparts in lower species like bacteria and yeast, for mitochondrial translation defects.

European Journal of Human Genetics (2011) 19, 394-399; doi:10.1038/ejhg.2010.214; published online 29 December 2010

Keywords: MRPS22; combined OXPHOS deficiency; mitochondrial translation; Cornelia de Lange-like phenotype

\section{INTRODUCTION}

Mitochondrial disorders are generally caused by dysfunction of the oxidative phosphorylation (OXPHOS) system. The OXPHOS system, comprising five enzyme complexes located in the mitochondrial inner membrane, is responsible for the production of most of the cell's ATP. Synthesis of this energy-generating system is controlled by both the mitochondrial and the nuclear genomes (mtDNA and nDNA). The mtDNA codes for 13 subunits of the OXPHOS complexes I, III, IV and $\mathrm{V}$ as well as the tRNAs and rRNAs required for the translation of these transcripts. All other mitochondrial proteins, including over 70 OXPHOS subunits and all proteins of the mitochondrial translation machinery, are encoded by nuclear genes. The majority of mutations associated with combined OXPHOS deficiencies because of impaired mitochondrial translation are located in mtDNA genes; ${ }^{1}$ however, the list of nDNA mutations is steadily growing with defects reported in mitochondrial translation factors, ${ }^{2-5}$ mitochondrial ribosomal proteins, ${ }^{6,7}$ mitochondrial tRNA synthetases ${ }^{8-10}$ and tRNA-modifying enzymes. ${ }^{11-13}$

We investigated a group of 33 patients with combined OXPHOS deficiencies (and normal complex II activities) by mutational analysis of the entire mtDNA, polymerase gamma and nuclear genes implicated in mitochondrial translation. In this study, we report a mutation in mitochondrial ribosomal protein MRPS22 detected in one patient and establish its pathogenicity.

\section{MATERIALS AND METHODS}

Case report

The male patient was the first child of healthy, first-grade consanguineous Pakistani parents. Fetal ultrasound disclosed microcephaly with dilatation of the third ventricle and a left ventricular hypertrophic cardiomyopathy (HCM). The patient was born by spontaneous vaginal delivery at 35 weeks of gestation with a birth weight of 2610 grams (P50), length $44 \mathrm{~cm}$ (P10) and head circumference $29 \mathrm{~cm} \quad(<\mathrm{P} 3)$. Physical examination showed Cornelia de Lange-like dysmorphic features (synophrys, low implanted posteriorly rotated ears, retrognathia, redundant skin over the neck, hypospadias, non-pitting edema of the limbs, back and eyelids, but no distal limb anomalies). Generalized muscle hypotonia and paucity of spontaneous movements were seen. Echocardiography showed biventricular and septal HCM with good function and pulmonary hypertension. MRI revealed corpus callosum hypoplasia and dilatation of the third ventricle, a subdural hematoma in the right fronto-parietal region, leukencephalopathy and delayed myelinisation. Chronic,

${ }^{1}$ Department of Pediatrics, Nijmegen Center for Mitochondrial Disorders, Radboud University Nijmegen Medical Center, Nijmegen, The Netherlands; ${ }^{2}$ Department of Genetics and Metabolic Diseases, Hadassah-Hebrew University Medical Center, Jerusalem, Israel; ${ }^{3}$ Department of Human Genetics, Radboud University Nijmegen Medical Center, Nijmegen, The Netherlands; ${ }^{4}$ University Hospital for Pediatric and Adolescent Medicine, Division of Inborn Metabolic Diseases, Heidelberg, Germany; ${ }^{5}$ Kinderneurologisches Zentrum SPZ, Allgemeinen Krankenhaus Hagen, Hagen, Germany; ${ }^{6}$ Department of Pediatrics, Academic Hospital Leuven, Leuven, Belgium

${ }^{*}$ Correspondence: Dr LP van den Heuvel, Department of Pediatrics, Nijmegen Center for Mitochondrial Disorders, Radboud University Nijmegen Medical Center, Geert Grooteplein 10, P.O. Box 9101, 6500 HB Nijmegen, The Netherlands. Tel: +31 24 3617983; Fax: +31 24 3618900; E-mail: B.vandenHeuvel@cukz.umcn.nl

7Joined first authorship.

Received 8 February 2010; revised 6 October 2010; accepted 10 November 2010; published online 29 December 2010 
severe metabolic acidosis with increased plasma lactate (max: $9 \mathrm{mmol} / \mathrm{l}$, $N:<2.1 \mathrm{mmol} / \mathrm{l})$, elevation of alanine in plasma $(684 \mu \mathrm{mol} / \mathrm{l}, N:<400 \mu \mathrm{mol} / \mathrm{l})$ and elevated urinary excretion of Krebs-cycle intermediates were noted. In the following months, dysphagia and failure to thrive necessitated gastrostomy. The biventricular HCM remained stable over the years with a shortening fraction of $39 \%$. ECG revealed Wolf-Parkinson-White syndrome. At the age of 2 years, he developed tonic seizures and EEG displayed a low background activity with multifocal sharp-slow waves. Seizures and EEG abnormalities disappeared spontaneously. Ophthalmological examination and hearing tests were repeatedly normal. Growth remained poor; growth parameters at the age of 4 years are as follows: weight $8.5 \mathrm{~kg}(<<<\mathrm{P} 3)$, length $80 \mathrm{~cm}(<<<\mathrm{P} 3)$, head circumference $40 \mathrm{~cm}(<<<\mathrm{P} 3)$. The facial features got less pronounced over the years. The boy hardly developed; now, at the age of 5.5 years, he shows a severe truncal hypotonia and tetraspasticity (left $>$ right) with sparse but dystonic movements. He is unable to sit freely and communicate, but he reacts to light and sounds.

\section{Cell culture}

Human skin fibroblasts were cultured in M199 medium (Gibco, Breda, The Netherlands) supplemented with $10 \%$ fetal calf serum (FCS) and antibiotics.

For complementation experiments, human skin fibroblasts were grown in DMEM (Dulbecco's modified Eagle's medium, Kibbutz Beit Haemek, Israel) with $4.5 \mathrm{~g} / \mathrm{l}$ of glucose, in the presence of $10 \%$ FCS, $50 \mu \mathrm{g} / \mathrm{ml}$ uridine and $110 \mu \mathrm{g} / \mathrm{ml}$ pyruvate. Selection of transfected cells was done in glucose-free RPMI (Roswell Park Memorial Institute) supplemented with $50 \mu \mathrm{m}$ galactose and $10 \%$ dialyzed FCS. ${ }^{14}$ Viability was assessed by trypan-blue exclusion.

\section{Enzyme measurements}

The activities of the OXPHOS enzyme complexes were measured in cultured skin fibroblasts as described previously; citrate synthase (CS) served as a control. ${ }^{15,16}$

\section{Pulse labeling of mitochondrial translation products}

In vitro labeling of mitochondrial translation was performed as described elsewhere, ${ }^{17}$ with a few minor adaptations. In short, cells were labeled for $60 \mathrm{~min}$ at $37^{\circ} \mathrm{C}$ in methionine-free DMEM with $10 \%$ dialyzed FCS, $200 \mu \mathrm{Ci} / \mathrm{ml}\left[{ }^{35} \mathrm{~S}\right]$ methionine ( $\operatorname{Tran}^{35} \mathrm{~S}$-Label, MP Biomedicals, Eindhoven, The Netherlands) and $100 \mu \mathrm{g} / \mathrm{ml}$ emetine, and subsequently chased for $10 \mathrm{~min}$ in regular DMEM with $10 \%$ FCS. Total cellular protein $(100 \mu \mathrm{g})$ was resuspended and incubated for $10 \mathrm{~min}$ in PBS containing 2\% lauryl maltoside. Unsolubilized material was removed by centrifugation at $10000 \mathrm{~g}$ for $10 \mathrm{~min}$ and loading buffer was added to the supernatant to a final concentration of $100 \mathrm{~mm}$ Tris-HCl (pH 6.8), 20\% glycerol, $1 \%$ SDS, $0.02 \%$ Coomassie Blue G-250 and $1 \%$ mercaptoethanol. Samples were run on $16 \%$ polyacrylamide gels, ${ }^{18}$ which were subsequently scanned on a FLA5100 (Fujifilm Life Science, Düsseldorf, Germany). Equal protein loading was confirmed by Coomassie blue staining.

\section{Homozygosity mapping}

Patient DNA was isolated from cultured skin fibroblasts by salt extraction. ${ }^{19}$ Whole-genome homozygosity mapping was performed using the GeneChip Human Mapping 10K Array Xba 142 version 2.0 (Affymetrix, High Wycombe, UK) in accordance with the manufacturer's guidelines. ${ }^{20}$ This array contains 10204 single neutral polymorphisms (SNPs) with a mean intermarker distance of $258 \mathrm{~kb}$, equivalent to $0.36 \mathrm{cM}$, and an average SNP heterozygosity of 0.38 .

\section{Sequence analysis}

Genomic DNA (gDNA) was extracted from cultured skin fibroblasts (patient) or blood (parents and controls) by standard procedures. ${ }^{19}$ Total RNA was isolated from patient fibroblasts using RNA-Bee (Tel-Test, Friendswood, TX, USA); complementary DNA (cDNA) was prepared using superscript II reverse transcriptase (Invitrogen, Breda, The Netherlands). Sequence analysis of MRPS22 (GenBank accession number NM_020191.2) was carried out on cDNA in two overlapping fragments; the parts of the first and last exon not covered, including the intron-exon boundaries, were amplified on gDNA. The presence of the mutation in exon 4 was confirmed on gDNA and subsequently screened for in both parents and 109 Pakistani controls. Sequence analysis of the entire open reading frame including the intron-exon junctions of NIPBL, SMC1A and SMC3 (GenBank accession numbers NM_015384.4, NM_005445.3 and NM_006306.2, respectively) was performed on gDNA. Primer sequences and PCR conditions used for amplification can be obtained upon request. Both strands were used for direct sequencing on a $3130 x l$ Genetic Analyzer, using the BigDye terminator v1.1 cycle sequencing kit according to the manufacturer's protocol (Applied Biosystems, Nieuwerkerk a/d IJssel, The Netherlands). Sequence data were analyzed with Sequencher 4.8 software (Gene Codes, Ann Arbor, MI, USA) by comparison with the reference sequences.

\section{Affymetrix NspI SNP array hybridization and analysis}

Copy number variation (CNV) screening by means of microarray analyses was carried out on the Affymetrix GeneChip 250k (NspI) SNP array platform (Affymetrix, Inc., Santa Clara, CA, USA), which contains 25-mer oligonucleotides representing a total of 262264 SNPs. Hybridizations were performed according to the manufacturer's protocols. Copy numbers were determined using the 2.0 version of the CNAG (Copy Number Analyzer for Affymetrix GeneChip mapping) software package, ${ }^{21}$ by comparing SNP probe intensities from patient DNA with those of a sex-matched pooled reference DNA sample (DNA from 10 healthy male individuals). The average resolution of this array platform, described by McMullan et al, ${ }^{22}$ is $150-200 \mathrm{~kb}$.

\section{Blue-native and SDS-PAGE analysis and in-gel activity assay}

Blue-native (BN) PAGE was used for separation of the OXPHOS complexes on $5-15 \%$ polyacrylamide-gradient gels as described before. ${ }^{23}$ After electrophoresis of $40 \mu \mathrm{g}$ mitochondrial protein from fibroblasts, gels were further processed for complex I in-gel activity assay and western blotting. Assembly of the OXPHOS complexes was investigated using monoclonal antibodies against subunits of complexes I, III, V (Molecular Probes, Leiden, The Netherlands), II and IV (MitoSciences, Eugene, OR, USA).

One-dimensional $10 \%$ SDS-PAGE analysis was performed as described previously. ${ }^{24}$ For detection of MRPS22, a polyclonal antibody raised against a GST-MRPS22 (full length) fusion protein (ProteinTech Group, Chicago, IL, USA) was used.

\section{Transfection with wild-type MRPS22 cDNA}

Wild-type MRPS22 cDNA was cloned into the pLenti6/V5-D-TOPO 6969-bp expression vector by directional TOPO cloning (primers and PCR conditions are available upon request). The recombinant vector was propagated in One Shot Stbl3 Escherichia coli and introduced into 293FT cells after co-transfection with pLP1, pLP2 and pLP/VSVG plasmids using lipofectamine according to the manufacturer's instructions (ViraPower, Invitrogen, Carlsbad, CA, USA). Control and patient cells were infected with the lentiviral construct carrying the wild-type MRPS22, and clones of stably transfected cells were established by blasticin $(4 \mu \mathrm{g} / \mathrm{ml})$ selection and by selection on glucose-free medium. ${ }^{7,25}$ Patient cells were also transiently transfected with the lentiviral vector carrying an expression control plasmid, pLenti6/V5-GW/lacZ (Invitrogen).

\section{Determination of complex IV levels}

Protein was determined by Bicinchoninic Acid (BCA) protein assay kit (SigmaAldrich, Rehovot, Israel). Amount of complex IV was estimated using the MitoProfile Biogenesis Dipstick assay kit (MitoSciences) according to the manufacturer's instructions. Results were obtained after digital imaging with TINA 2.0 software.

\section{Determination of mitochondrial $12 \mathrm{~S}$ rRNA and $16 \mathrm{~S}$ rRNA levels}

Total mRNA isolated using TRI reagent (Sigma-Aldrich) was reverse transcribed with ImProm-II reverse transcriptase (Promega, Madison, WI, USA). The cDNA was amplified in a PCR reaction to detect the expression of specific nucleic acid sequences using SYBR Green (Applied Biosystems, Roche, Branchburg, NJ, USA). $\beta$-Actin served as an endogenous control and mitochondrial $12 \mathrm{~S}$ rRNA or $16 \mathrm{~S}$ rRNA as the target cDNAs (primers are available on request). The levels of all cDNAs were equalized and real-time PCR reactions were performed using each primer set separately. The relative expression of the 
target transcript was calculated with the comparative Ct method (Applied Biosystems User Manual) and analyzed by two-tailed Student's $t$-test.

\section{RESULTS}

Enzyme activities of OXPHOS complexes I, III and IV in patient fibroblasts were reduced to 45,59 and $36 \%$ of the lowest control value, respectively; complex II was not affected (Table 1). BN-PAGE analysis confirmed this combined OXPHOS deficiency: complex I and IV were hardly detectable and complex III showed a slight decrease in the amount of fully assembled complex, whereas the level of complex II was normal (Figure 1). Additionally, complex I activity was again clearly lowered.

Pulse labeling of mitochondrial protein synthesis products revealed a marked and generalized mitochondrial translation defect (Figure 2): total incorporation of $\left[{ }^{35} \mathrm{~S}\right]$ methionine was $26 \%$ of that in controls. The decrease in mitochondrial translation varied between different subunits, with the translation rates of ND5, ND6 and COIII/COII (10-15\%) being most severely and of ATP8 and ND4L/ATP6 (60-70\%) being the least affected.

After excluding mutations in the mtDNA, polymerase gamma (POLG and POLG2) and all nine known mitochondrial translation factors (MTIF2, MTIF3, TSFM, TUFM, GFM1, GFM2, MTRF1, $M T R F 1 L$ and $M R R F$ ) by direct sequencing, we used homozygosity mapping to guide further investigations. Multiple homozygous regions were found, containing mitochondrial ribosomal proteins, among others. On the basis of the mitochondrial translation deficit in patient fibroblasts and previously reported mutations, we selected MRPS16 (located in the largest homozygous region, spanning $110.9 \mathrm{Mb}$ on chromosome 10) and MRPS22 (located in a region of $16.1 \mathrm{Mb}$ between SNPs rs977683 and rs1112189 on chromosome 3) as plausible candidate genes. Sequence analysis revealed a homozygous $\mathrm{T}$ to $\mathrm{C}$ transition at position 644 of the coding sequence of MRPS22, which is predicted to result in the substitution of the highly conserved leucine for proline at position 215 (Figure $3 \mathrm{a}$ and $\mathrm{b}$ ). The mutation was heterozygous in both parents and absent in 109 ethnically matched controls. As MRPS22 has no ortholog in bacteria and no crystal structure exists for modeling, we used the PSIPRED server to predict the secondary structure of the MRPS22 protein. ${ }^{26}$ The mutation seems to be located in the middle of a helix (Figure 3c) and presumably disturbs it because of the lack of stabilizing hydrogen bond formation by proline and the possible introduction of a kink. The homology-based tools PolyPhen (Polymorphism Phenotyping, http://genetics.bwh.harvard.edu/pph/ index.html) ${ }^{27}$ and SIFT (Sorting Intolerant From Tolerant, http://sift.jcvi.org/ $)^{28}$ were applied to indicate the functional impact of the missense mutation. Both PolyPhen and SIFT predicted the mutation to be deleterious (with a PSIC score difference of 2.243 and a score of 0.00 , respectively). Western blotting revealed the (near) absence of MRPS22 in patient fibroblasts (Figure 3d).

To investigate whether the MRPS22 deficit causes the OXPHOS system deficiency, we introduced wild-type MRPS22 cDNA into patient cells. The complex IV enzyme activity increased from $40 \%$ to $109-118 \%$ and its protein level went from $51 \%$ to $116-120 \%$ of the control values (Table 2). Quantification of mitochondrial rRNA levels showed a restoration of $12 \mathrm{~S}$ and $16 \mathrm{~S}$ rRNA content from 36 and $77 \%$ to $71-$ $109 \%$ and $70-120 \%$ of control levels (Figure $4 \mathrm{a}$ and b), respectively, resulting in normal $12 \mathrm{~S}$ rRNA/16S rRNA ratios (Figure 4c). Complex IV activity and amount, as well as rRNA content, were decreased after transfection of patient fibroblasts with a control vector.

Subsequently, genes known to be implicated in Cornelia de Lange syndrome were screened for mutations to shed light on the genetic origin of the Cornelia de Lange-like dysmorphic features. No
Table 1 OXPHOS enzyme activities in fibroblasts

\begin{tabular}{|c|c|c|}
\hline \multirow[b]{2}{*}{ Complex } & \multicolumn{2}{|c|}{ Activities (mU/U citrate synthase) $)^{\mathrm{a}, \mathrm{b}}$} \\
\hline & Patient & Reference range \\
\hline I & 45 & $100-310$ \\
\hline II & 606 & $520-845$ \\
\hline III & 782 & 1320-2610 \\
\hline$|I+I I|$ & 186 & $110-470$ \\
\hline IV & 242 & $680-1190$ \\
\hline
\end{tabular}

aMeasured in mitochondrial-enriched fractions from fibroblasts.

bThe activity of citrate synthase was $208 \mathrm{mU} / \mathrm{mg}$ protein (reference range $144-257 \mathrm{mU} / \mathrm{mg}$ protein).

Decreased activities as compared with controls are shown in bold numbers.

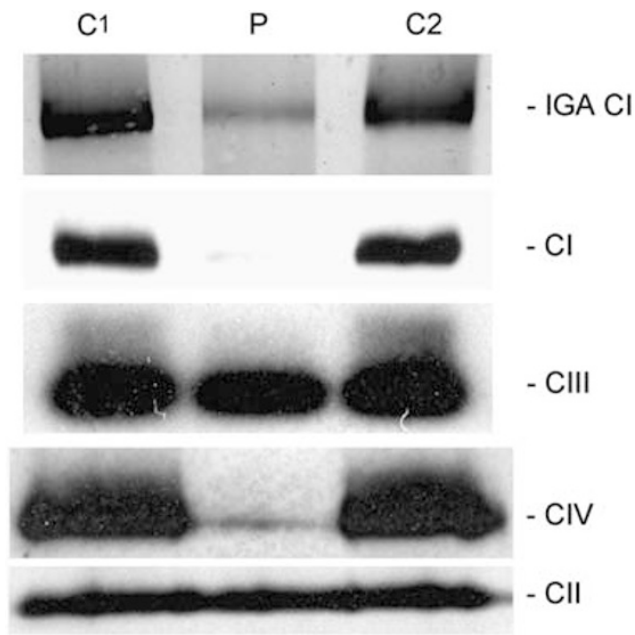

Figure 1 BN-PAGE analysis in patient fibroblasts. Antibodies directed against specific individual subunits were used to assess the amount of each of the fully assembled complexes in the patient (P) and controls (C1 and C2). Complex II was used as a loading control. Cl-CIV=complex I-IV; IGA=in-gel activity.

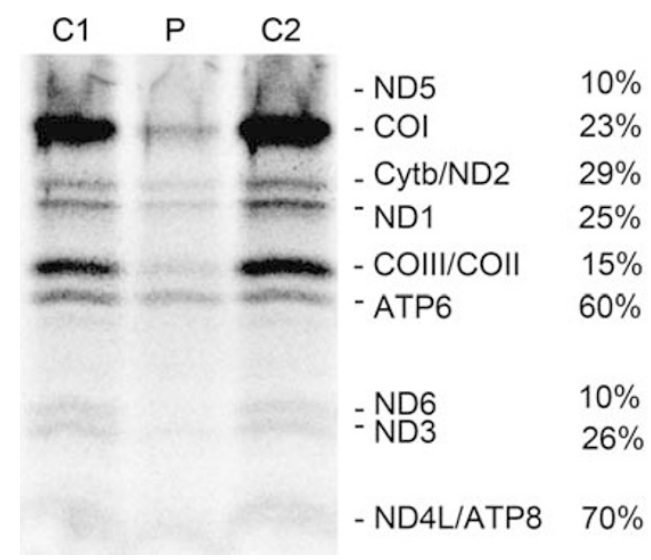

Figure 2 In vitro labeling of mitochondrial translation products. The patient (P) translation rates of the different subunits are expressed as percentages of the average $\left.{ }^{35} \mathrm{~S}\right]$ methionine incorporation in two controls ( $\mathrm{C} 1$ and $\mathrm{C} 2$ ). The level of translation of ND5 was obtained from an identical assay, in which this product was clearly visible. COI-COIII=subunits of cytochrome $c$ oxidase (complex IV); cyt $\mathrm{b}=$ cytochrome $b$ subunit (of complex III); ND1-6=subunits of NADH CoQ oxidoreductase (complex I); ATP6-8=ATP synthase (complex V) subunits 6 and 8 . 


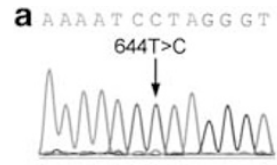

Patient

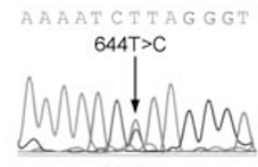

Mother

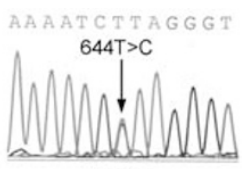

Father b

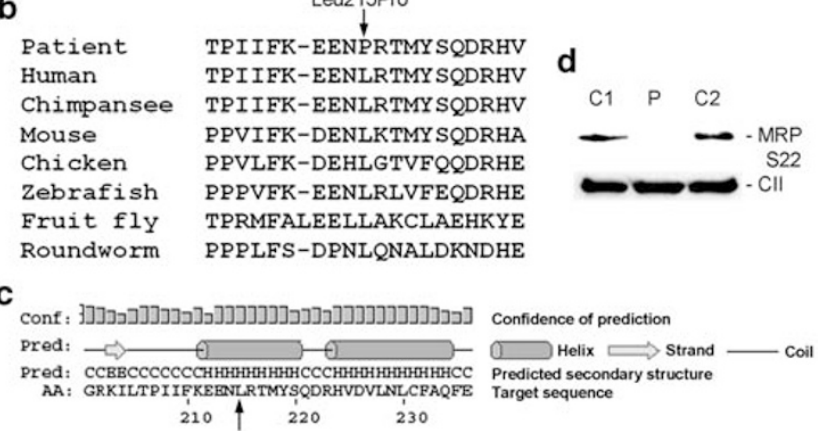

Figure 3 MRPS22 mutation, conservation of the mutated residue, protein structure prediction and protein level in patient cells. (a) DNA sequence analysis of the patient (homozygous c.644T $>$ C) and both parents (heterozygous). (b) Protein sequence alignment from human to worm, which shows that the mutated leucine residue is highly conserved among different species. (c) Prediction of the MRPS22 protein structure, displaying part of the protein with the Leu215Pro substitution located in the middle of a helix. (d) Immunoblot analysis in patient (P) and control (C1 and C2) fibroblasts reveals the (near) absence of the MRPS22 protein. Complex II was used as a loading control.

Table 2 Restoration of complex IV activity and amount by MRPS22 transfection

\begin{tabular}{|c|c|c|c|}
\hline \multirow[b]{2}{*}{ Sample } & \multicolumn{2}{|c|}{ Activities (mU/U citrate synthase) ${ }^{a}$} & \multirow{2}{*}{$\begin{array}{l}\text { Amount } \\
\text { Complex IV }\end{array}$} \\
\hline & Complex IV & Complex II & \\
\hline Control & 2230 & 190 & 2.49 \\
\hline Control+MRPS22 & 1780 & 190 & 3.35 \\
\hline Patient & 890 & 230 & 1.26 \\
\hline Patient+MRPS22(1) ${ }^{\mathrm{c}}$ & 2440 & 190 & 2.88 \\
\hline Patient+MRPS22(2) ${ }^{\mathrm{c}}$ & 2630 & 250 & 2.99 \\
\hline Patient+lacZ & 650 & 240 & 0.40 \\
\hline
\end{tabular}

aMeasured in fibroblast homogenates.

${ }^{b}$ Expressed relative to frataxin.

'Stably transfected Patient+MRPS22 clones 1 and 2

mutations were found in the genes NIPBL, SMC1A and SMC3. In addition, analysis of patient DNA on the 250K NspI SNP array revealed a normal male profile, thus excluding any potentially clinically relevant copy number variants.

\section{DISCUSSION}

This study confirms the importance of mitochondrial ribosomal protein MRPS22 for mitochondrial translation and consequently for the formation and functioning of the OXPHOS system. We described the second mutation in MRPS 22 and determined its pathogenic role in the patient's mitochondrial disorder.

The combined OXPHOS complex I, III and IV deficiency, impairment of mitochondrial translation and absence of mutations in the mtDNA in our patient suggested that the genetic defect was located in a nuclear gene encoding a constituent of the mitochondrial translation machinery. Sequence analysis of all mitochondrial translation factors followed by homozygosity mapping led to the identification of a homozygous missense mutation in MRPS22, predicted to substitute a highly conserved leucine for proline (Leu215Pro). In patient fibroblasts, the MRPS22 protein was undetectable and expressing the wild-type MRPS22 cDNA rescued the disease phenotype, evinced by the restoration of OXPHOS complex IV amount and activity, as well as $12 \mathrm{~S}$ rRNA transcript level, establishing the MRPS22 defect as the cause of the disease.

Human MRPS22 is a 360-amino-acid protein that has been found in a limited number of species, having no ortholog in fungi or prokaryotes. ${ }^{29}$ The exact location of MRPS22 in the three dimensional structure of the ribosome and its exact function are currently unknown. Recently, a MRPS22 defect was shown to strongly hamper assembly of the small ribosomal subunit, whereas assembly of the large subunit and part of the small subunit were only mildly affected. ${ }^{30}$ This is consistent with both our and previous findings that mutations in mitochondrial ribosomal small subunit proteins cause a marked decrease in the abundance of the $12 \mathrm{~S}$ rRNA transcript and nearly no reduction in the level of $16 \mathrm{~S}$ rRNA, ${ }^{6,7}$ as rRNAs are readily degraded unless they are incorporated into a ribosomal subunit. ${ }^{31}$ Thus, although MRPS22 is evolutionary not well conserved, it is indispensable for the assembly of the human mitochondrial ribosome and consequently, as we have shown, for effective mitochondrial protein synthesis. Previously, a missense mutation in MRPS22 (Arg170His) ${ }^{7}$ and a non-sense mutation in MRPS16 $(\operatorname{Arg} 111 \mathrm{X}),{ }^{6}$ which is highly conserved and essential for ribosome assembly, ${ }^{29,30,32}$ were reported to be lethal. The clinical phenotypes of patients with MRPS16 or MRPS22 mutations bear resemblances. All three mutations are associated with muscle hypotonia, lactic acidemia and edema of the limbs. Furthermore, both MRPS22 defects led to hypertrophic cardiomyopathy. The patient harboring the MRPS22 Arg170His mutation additionally presented with tubulopathy and no brain abnormalities were noted, in contrast to the patient reported in this study. It remains unclear whether the brain abnormalities observed in our patient are primarily due to the genetic defect or developed secondary to intrauterine ischemia; presumably both contributed. The dysmorphic features and brain abnormalities of our patient are similar to those seen in the MRPS16 patient, however, the latter did not develop hypertrophic cardiomyopathy. On the basis of the identification of a MRPS22 mutation and the absence of mutations or copy number variations responsible for the Cornelia de Lang-like symptoms in our patient, we advise to perform echocardiography, metabolic screening (plasma lactate, plasma amino acid analysis and urinary organic acid analysis) and cerebral MRI in patients with dysmorphic features suggestive of Cornelia de Lange syndrome. Notably, in our patient these features were quite prominent at birth but diminished over the years.

The patient reported in this study was part of a cohort of 33 patients with combined OXPHOS deficiencies. In seven other patients from this cohort, the causative genetic defect was found. Mutations were identified in the mitochondrial tRNA ${ }^{\text {Lys }}, \mathrm{tRNA}^{\mathrm{Arg}}$ and $\mathrm{tRNA}^{\mathrm{Trp}}$ genes (four patients), ${ }^{33}$ POLG (one patient) and translation factors TSFM and GFM1 (two patients), including three mutations that had not been published before. Our findings emphasize that nuclear gene mutations contribute substantially to mitochondrial translation deficits. Vastly exceeding 100 proteins, among which 81 mitochondrial ribosomal proteins, ${ }^{29}$ are (in)directly involved in the mitochondrial translation process, whereas in merely nine of these proteins mutations have been found up till now. Therefore, many more such defects are expected to be discovered. Progress in high-throughput sequencing technologies will hopefully make the difficult task of selecting the right candidate genes obsolete. In the meantime, supernumerary proteins 

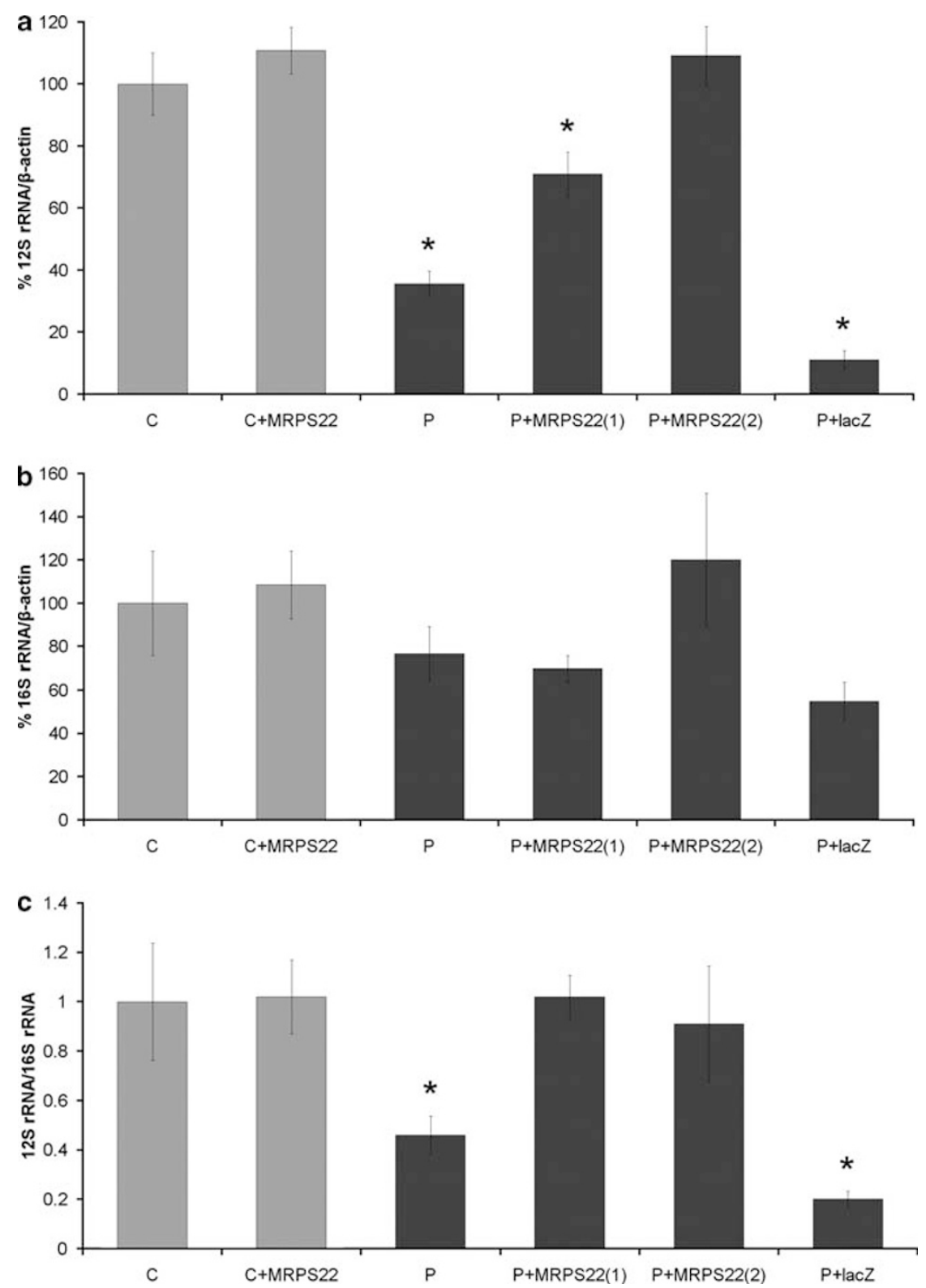

Figure 4 Restoration of mitochondrial rRNA levels by MRPS22 transfection. Patient $(\mathrm{P})$ and control $(\mathrm{C})$ fibroblasts were transfected with a viral vector carrying the wild-type MRPS22 cDNA. Stably transfected clones (C+MRPS22, P+MRPS22(1) and P+MRPS22(2)) were isolated by antibiotic selection. Patient cells were also transfected with a control vector (lacZ). 12S rRNA and 16S rRNA levels were quantified relative to $\beta$-actin and presented relative to control values as mean percentages \pm SD $(\mathbf{a}, \mathbf{b})$ or as the relative ratios of $12 \mathrm{~S}$ rRNA:16S rRNA \pm SD $(\mathbf{c})$ of triplicate determinations. * Significantly different from the control $(P<0.05)$.

such as MRPS22, lacking homologs in bacteria and other lower species, should not be disregarded during investigations of mitochondrial disorders as they evidently perform important roles.

\section{CONFLICT OF INTEREST}

The authors declare no conflict of interest.

\section{ACKNOWLEDGEMENTS}

This work was supported by the European Union's Sixth Framework Program, contract number LSHMCT-2004-005260 (MITOCIRCLE), and in part by the Association Française contre les Myopathies (AFM) and the Israeli Ministry of Health to AS. We thank Ilse Pronk and Benigna Geurts (Nijmegen Center for
Mitochondrial Disorders) for their work on the sequence analysis of MRPS22 and Hanka Venselaar (Center for Molecular and Biomolecular Bioinformatics, Nijmegen) for the secondary structure prediction. Maleeha Azam and Dr Raheel Qamar (COMSATS Institute of Information Technology, Islamabad) are acknowledged for providing the Pakistani control samples. We are grateful to Dr Michael Marusich (MitoSciences, OR, USA) for supplying the MitoProfile assay kit. Finally, Dr Avraham Shaag (Hadassah-Hebrew University Medical Center, Jerusalem) is acknowledged for helpful comments regarding the rRNA quantification.

1 MITOMAP: A Human Mitochondrial Genome Database. http://www.mitomap.org, 2009. 
2 Antonicka H, Sasarman F, Kennaway NG, Shoubridge EA: The molecular basis for tissue specificity of the oxidative phosphorylation deficiencies in patients with mutations in the mitochondrial translation factor EFG1. Hum Mol Genet 2006; 15: 1835-1846.

3 Coenen MJ, Antonicka H, Ugalde $\mathrm{C}$ et al: Mutant mitochondrial elongation factor G1 and combined oxidative phosphorylation deficiency. N Engl J Med 2004; 351: 2080-2086.

4 Smeitink JA, Elpeleg O, Antonicka $\mathrm{H}$ et al: Distinct clinical phenotypes associated with a mutation in the mitochondrial translation elongation factor EFTs. Am J Hum Genet 2006; 79: 869-877.

5 Valente L, Tiranti V, Marsano RM et al: Infantile encephalopathy and defective mitochondrial DNA translation in patients with mutations of mitochondrial elongation factors EFG1 and EFTu. Am J Hum Genet 2007; 80: 44-58.

6 Miller C, Saada A, Shaul N et al: Defective mitochondrial translation caused by a ribosomal protein (MRPS16) mutation. Ann Neurol 2004; 56: 734-738.

7 Saada A, Shaag A, Arnon S et al: Antenatal mitochondrial disease caused by mitochondrial ribosomal protein (MRPS22) mutation. J Med Genet 2007: 44: 784-786.

8 Edvardson S, Shaag A, Kolesnikova 0 et al: Deleterious mutation in the mitochondrial arginyl-transfer RNA synthetase gene is associated with pontocerebellar hypoplasia. Am J Hum Genet 2007; 81: 857-862.

9 Scheper GC, van der Klok T, van Andel RJ et al: Mitochondrial aspartyl-tRNA synthetase deficiency causes leukoencephalopathy with brain stem and spinal cord involvement and lactate elevation. Nat Genet 2007; 39: 534-539.

10 Isohanni P, Linnankivi T, Buzkova $\mathrm{J}$ et al: DARS2 mutations in mitochondrial leukoencephalopathy and multiple sclerosis. J Med Genet 2010; 47: 66-70.

11 Bykhovskaya Y, Casas K, Mengesha E, Inbal A, Fischel-Ghodsian N: Missense mutation in pseudouridine synthase 1 (PUS1) causes mitochondrial myopathy and sideroblastic anemia (MLASA). Am J Hum Genet 2004; 74: 1303-1308.

12 Fernandez-Vizarra E, Berardinelli A, Valente L, Tiranti V, Zeviani M: Nonsense mutation in pseudouridylate synthase 1 (PUS1) in two brothers affected by myopathy, lactic acidosis and sideroblastic anaemia (MLASA). J Med Genet 2007; 44: 173-180.

13 Zeharia A, Shaag A, Pappo 0 et al: Acute infantile liver failure due to mutations in the TRMU gene. Am J Hum Genet 2009; 85: 401-407.

14 Robinson $\mathrm{BH}$ : Use of fibroblast and lymphoblast cultures for detection of respiratory chain defects. Methods Enzymol 1996; 264: 454-464.

15 Janssen AJ, Smeitink JA, van den Heuvel LP: Some practical aspects of providing a diagnostic service for respiratory chain defects. Ann Clin Biochem 2003; 40: 3-8.

16 Smeitink J, Sengers R, Trijbels F, van den Heuvel L: Human NADH:ubiquinone oxidoreductase. J Bioenerg Biomembr 2001; 33: 259-266.

17 Boulet L, Karpati G, Shoubridge EA: Distribution and threshold expression of the tRNA(Lys) mutation in skeletal muscle of patients with myoclonic epilepsy and raggedred fibers (MERRF). Am J Hum Genet 1992; 51: 1187-1200.
18 Schagger H, von Jagow G: Blue Native electrophoresis for isolation of membrane protein complexes in enzymatically active form. Anal Biochem 1991; 199: 223-231.

19 Miller SA, Dykes DD, Polesky HF: A simple salting out procedure for extracting DNA from human nucleated cells. Nucleic Acids Res 1988; 16: 1215.

20 Matsuzaki H, Loi H, Dong S et al: Parallel genotyping of over 10000 SNPs using a one-primer assay on a high-density oligonucleotide array. Genome Res 2004; 14: 414-425.

21 Nannya $Y$, Sanada M, Nakazaki $\mathrm{K}$ et al: A robust algorithm for copy number detection using high-density oligonucleotide single nucleotide polymorphism genotyping arrays. Cancer Res 2005; 65: 6071-6079.

22 McMullan DJ, Bonin M, Hehir-Kwa JY et al: Molecular karyotyping of patients with unexplained mental retardation by SNP arrays: a multicenter study. Hum Mutat 2009; 30: 1082-1092.

23 Calvaruso MA, Smeitink J, Nijtmans L: Electrophoresis techniques to investigate defects in oxidative phosphorylation. Methods 2008; 46: 281-287.

24 Ugalde C, Vogel R, Huijbens R, van den Heuvel B, Smeitink J, Nijtmans L: Human mitochondrial complex I assembles through the combination of evolutionary conserved modules: a framework to interpret complex I deficiencies. Hum Mol Genet 2004; 13 : 2461-2472.

25 Saada A, Edvardson S, Rapoport $M$ et al: C60RF66 is an assembly factor of mitochondrial complex I. Am J Hum Genet 2008; 82: 32-38.

26 Jones DT: Protein secondary structure prediction based on position-specific scoring matrices. J Mol Biol 1999; 292: 195-202.

27 Ramensky V, Bork P, Sunyaev S: Human non-synonymous SNPs: server and survey. Nucleic Acids Res 2002; 30: 3894-3900.

28 Ng PC, Henikoff S: SIFT: Predicting amino acid changes that affect protein function. Nucleic Acids Res 2003; 31: 3812-3814.

29 Smits P, Smeitink JA, van den Heuvel LP, Huynen MA, Ettema TJ: Reconstructing the evolution of the mitochondrial ribosomal proteome. Nucleic Acids Res 2007; 35: 4686-4703.

30 Emdadul HM, Grasso D, Miller C, Spremulli LL, Saada A: The effect of mutated mitochondrial ribosomal proteins S16 and S22 on the assembly of the small and large ribosomal subunits in human mitochondria. Mitochondrion 2008; 8: 254-261.

31 Dennis PP, Young RF: Regulation of ribosomal protein synthesis in Escherichia coli B/r. J Bacteriol 1975; 121: 994-999.

32 Cavdar KE, Burkhart W, Blackburn K, Moseley A, Spremulli LL: The small subunit of the mammalian mitochondrial ribosome. Identification of the full complement of ribosomal proteins present. J Biol Chem 2001; 276: 19363-19374.

33 Smits P, Mattijssen S, Morava E et al: Functional consequences of mitochondrial tRNA Trp and tRNA Arg mutations causing combined OXPHOS defects. Eur J Hum Genet 2010; 18: 324-329. 